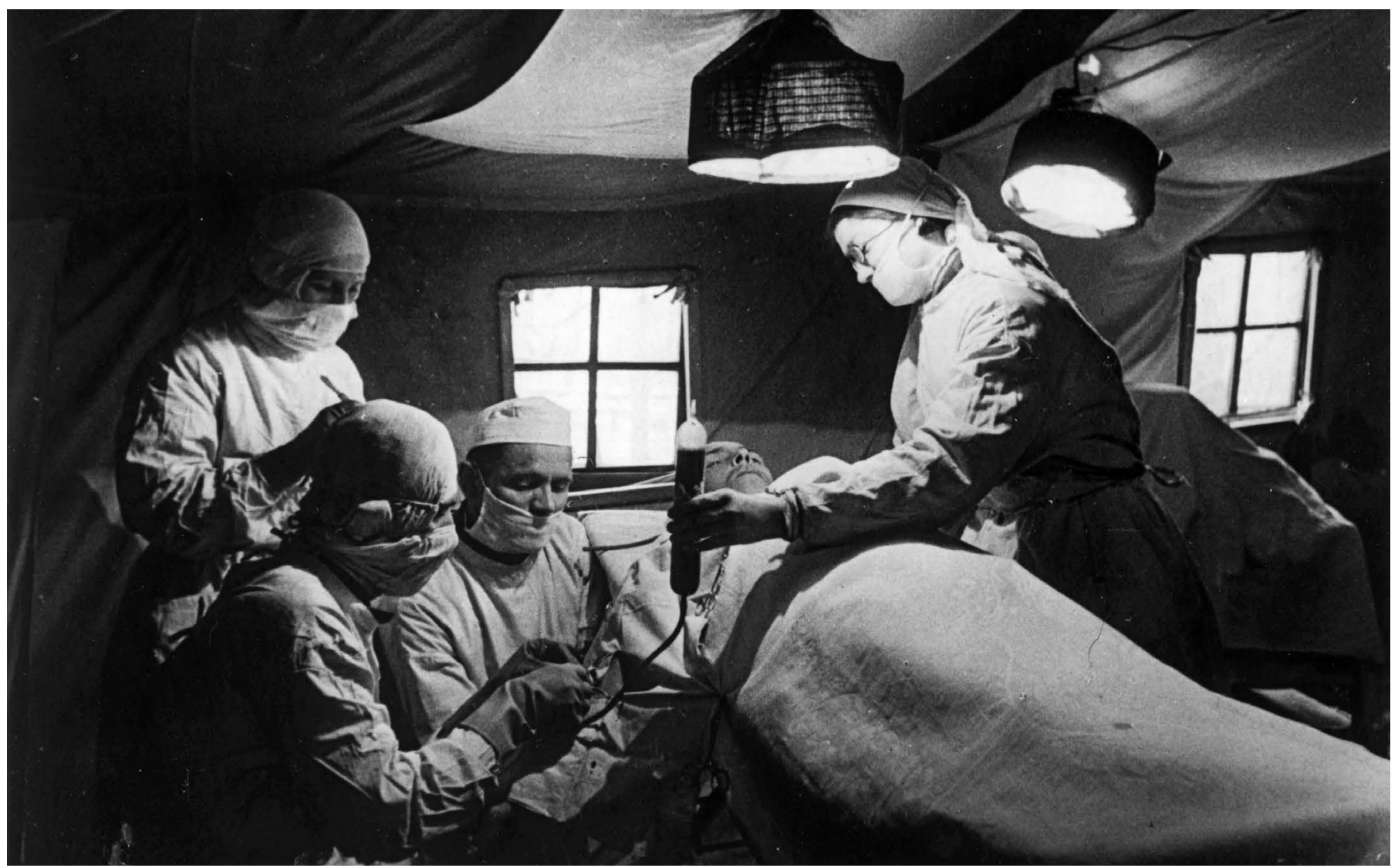

A blood transfusion is conducted in a field hospital on the Russian Western Front during the Second World War.

\title{
TRANSFUSION
}

\section{Too much of a good thing}

\section{The ability to give donated blood to patients has saved countless lives. But the routine nature of such transfusions is being rethought.}

\section{BY BIANCA NOGRADY}

$\mathrm{F}$ Ior a medical treatment to be approved by regulatory bodies, it must go through a rigorous sequence of laboratory testing, studies in animals and clinical trials to establish its safety and efficacy.

Yet there is a centuries-old treatment used every day in intensive-care units and emergency departments worldwide that, until two decades ago, had never been put through the large-scale studies that evidence-based medicine demands.

The procedure in question is blood transfusion. Since the early 1800 s, when the first recorded human-to-human transfusion was conducted, saving a woman who was bleeding heavily after giving birth, transfusions have become a mainstay of critical-care medicine.

But the long-held consensus on transfusion may not, in fact, be best practice. To begin with, people with certain conditions who receive blood might do just as well without it.
There is also a suggestion that transfused blood itself - aside from the associated risks of infection or rejection - may have immunological and physiological effects on the body that are not necessarily benign. "People have been practicing transfusion for a long time but we didn't always have the good-quality evidence base to help us to know how to do it," says Erica Wood, a specialist in transfusion medicine at Monash University in Melbourne, Australia.

More than 30 clinical trials, covering a range of patient groups and medical scenarios, have examined the effects of providing transfusions under only the most urgent of circumstances, as measured by the level of oxygen-carrying protein haemoglobin in the patient's blood. These trials overwhelmingly have found no difference in death rate - or in other outcomes such as stroke, sepsis, kidney failure, pneumonia or wound infection - between transfusing blood to the patient when the haemoglobin level drops below a 'liberal' threshold of 9-10 grams of haemoglobin per decilitre or a more 'restrictive' threshold of 7-8 grams per decilitre ${ }^{1}$.

\section{CHANGING THE DEFAULT}

A rethink of the transfusion threshold began in 1999 with a trial that looked at transfusions in critically ill people ${ }^{2}$, set against a backdrop of increasing concern about blood-borne diseases and the cost of transfusions. This pivotal study, involving 838 intensive-care patients in Canada, found that transfusing blood only when a patient's haemoglobin level dropped to 7 grams per decilitre - as opposed to below 10 grams per decilitre, which had been the standard since the 1940s - would not increase the risk of poor outcomes.

The death rate from all causes within 30 days of patients' admission to intensive care was similar regardless of whether the decision to transfuse blood was implemented at the higher or lower haemoglobin level. In fact, patients who were given a transfusion under the 
more-restrictive threshold were less likely to die during their hospital stay, and had slightly lower levels of organ dysfunction.

The Canadian trial marked a turning point for transfusion medicine, says Jeffrey Carson, an internist at the Robert Wood Johnson Medical School at Rutgers University in New Jersey. "If you don't transfuse, you can't get side effects from blood," he says. "If it doesn't improve outcomes, there's no good reason to use more blood."

Wood says clinicians are now focusing on why someone might need blood - for example, being referred to the emergency department with severe anaemia - and addressing that reason directly.

"Many people are safely treated with intravenous iron, rather than transfusion," she says. "If we give them somebody else's red blood cells, it might increase their haemoglobin today, but those red blood cells will not last a long time - and you haven't fixed their problem, which is a lack of iron."

The re-evaluation of transfusions is also changing surgical practice. James Isbister, a haematologist at the Sydney Medical School, says that, at present, most transfusions are given on a prophylactic basis. "The doctor is either expecting a problem or wants to cover themselves for something that may or may not happen," he notes.

Instead of relying on a post-surgery blood transfusion to prevent anaemia, clinicians are working to identify and address anaemia in patients long before they go under the knife, as well as taking steps to minimize blood loss during operations.

"When you look through the alternatives to transfusion, most of them are, in fact, good clinical medicine," Isbister says. "The message is transfusion should not be a default decision until you've really worked out what the problem is and the best way to treat it."

There are also people for whom a blood transfusion is truly life-saving: those who have lost huge amounts of blood owing to trauma or to bleeding during childbirth; those with conditions affecting the red blood cells, such as sickle-cell anaemia or thalassaemia; and those whose bone marrow has been depleted by chemotherapy. "There are a lot of times when blood is really needed for life-saving," says Majed Refaai, a pathologist at the University of Rochester Medical Center in New York. But he objects to transfusions being given simply to raise a patient's haemoglobin level.

\section{THE THRESHOLD QUESTION}

Such thinking is becoming the norm. Indeed, guidelines for when to perform transfusions have become more restrictive worldwide, including those used in the United States, Australia, the United Kingdom and much of Europe. But a restrictive threshold and a lower level of haemoglobin may not be suitable for all patients.

One such group comprises people who have experienced a heart attack or who are undergoing heart surgery, says Carson. He points to a trial in people receiving heart surgery that suggested the more restrictive transfusion threshold was actually associated with a slightly higher rate of mortality ${ }^{3}$. Given that a heart attack is the result of a blockage in the arteries supplying blood - and therefore oxygen - to the muscles of the heart, it stands to reason that boosting the blood supply could help to limit the damage, Carson says.

"The nature of oxygen

"If you don't transfuse, you can't get side effects from blood.' metabolism in the heart is that it extracts a large percentage of the oxygen that a red blood cell delivers - much higher than other parts of the body," he says.

Carson and colleagues conducted a pilot study ${ }^{4}$ in 110 people being treated for a heart attack, which hinted at better outcomes when a liberal transfusion threshold of 10 or more grams of haemoglobin per decilitre was used, and they are now embarking on a follow-up study in 3,500 people. Similar concerns exist for people who have experienced injury to the brain - another organ that is acutely sensitive to reduced blood flow and oxygen levels.

A group of diseases that might also benefit from a more liberal threshold for transfusion are the blood cancers: leukaemia, myeloma and lymphoma. Zoe McQuilten, a haematologist at Monash University, says that people with conditions that require regular transfusions are very different from the acutely ill patients who are most often the subject of trials on transfusion.

For one thing, they are not in hospital and are living close to normal lives - regular transfusions notwithstanding - yet they are assessed using the same haemoglobin thresholds as people in intensive care, McQuilten says. An upcoming feasibility study hopes to address this by taking a different approach to the question. Instead of exploring whether a restrictive threshold is as safe as a liberal threshold in the critically ill, "we're asking would a higher haemoglobin level result in a higher quality of life in patients who are chronically transfused," she says.

\section{DO NO HARM}

There is also the long-standing question of whether transfusions are entirely benign. Aside from potential issues of contamination and severe immune reactions, there is the possibility that the transfused blood itself could be causing harm.

Refaai argues that because blood is essentially a 'liquid' organ, a transfusion is equivalent to an organ transplant, with its attendant risks. He points to growing evidence that, under the same circumstances, patients who undergo the same procedure with the same risk factors actually do better if they don't have a transfusion compared to if they do.

For example, transfusions have been associated with an increased risk of infection in recipients. McQuilten says this could be explained by the iron hypothesis, which states that older red blood cells are more likely to be broken down than fresh ones in the first few hours following transfusion. This is thought to lead to the release of haemoglobin and iron from the damaged cells into the extracellular space that, in turn, can promote the growth of bacteria.

Another concern is that storage could have adverse effects on blood. Blood products comprising red blood cells typically have a shelf life of up to 42 days. But Jamie Cooper, a criticalcare physician and director of the Australian and New Zealand Intensive Care Research Centre at Monash University, says that red blood cells may undergo structural and biochemical changes during storage that could have harmful consequences for recipients. For example, instead of retaining a round, smooth and flexible disc-like shape, older red blood cells become more rigid and pointed or spiky, which is also known as spiculation.

"We're worried that spiculated older cells might not travel so well through the microcirculation," Cooper says. "This might be a problem with critically ill patients who have shock, as they have lots of changes in microcirculation."

To make matters even more complicated, there's the possibility that extremely fresh blood may also make patients more vulnerable to acquiring outside infections compared to blood of an intermediate age. Cooper says that there might be a 'sweet spot' for the age of stored blood: a storage duration that minimizes the possible risks of both fresh and older red blood cells. "It could be that a red cell in a bag is like a good Chianti; it has to sit there for a while and develop a bit of age."

Several large studies have assigned patients randomly to receive transfusions of either fresh or older blood, with mixed results. Cooper says the challenge is to conduct a study large enough to detect what could be small differences in outcomes relating to the age of blood.

"It is very important, because it's so critical that blood transfusion is safe," Cooper says. Given the frequency of blood transfusions, and the vulnerability of those receiving them, there is little room for uncertainty.

"If a blood transfusion was bad for you, even a tiny bit," says Cooper, "it would be better if we were more judicious."

Bianca Nogrady is a freelance science writer in Sydney, Australia.

1. Carson, J. L. et al. Cochrane Database Syst. Rev. 10, CD002042 (2016).

2. Hébert, P. C. et al. N. Engl. J. Med. 340, 409-417 (1999).

3. Murphy, G. M. et al. N. Engl. J. Med. 372, 997-1008 (2015).

4. Carson, J. L. et al. Am. Heart J. 165, 964-971 (2013). 